\title{
Glass Breaking in Fires
}

PATRICK J. PAGNI and ARUNA A. JOSHI

Mechanical Engineering Department

University of California

Berkeley, California 94720, USA

\begin{abstract}
Glass breaking in compartment fires is an important practical problem since a window acts as a wall before breaking and as a vent after breaking. If sufficient excess pyrolyzates have accumulated in the hot layer, this sudden geometric change can lead to backdraft and flashover. As Emmons explained at the First Symposium, windows break in fires due to thermal stress from the differential heating of the central portion and the shaded edge. The focus of this paper is on quantifying the connection between the compartment fire and the glass temperature to predict the window breaking time, $t_{b}$. Techniques are presented for accurately calculating the history of the central glass temperature profile, $T(x, t)$, for any fire exposure. Two-dimensional temperature histories, $T(x, y, t)$, where $x$ is depth and $y$ is toward the center, and mean stress histories, $\sigma_{z z}(y, t)$, are also calculated. It is determined here that breaking occurs when the mean glass temperature difference is $\Delta T=\left(\sigma_{b} / E \beta\right) g$, where $\sigma_{b} / E$ is the maximum glass tensile strain, $\beta$ is the thermal coefficient of linear expansion and $g$ is a geometry factor of order one. Calculations suggest that the edge remains at its initial temperature, $T_{i}$, so that $\Delta T=T\left(t_{b}\right)-T_{i}$, when the shading is large, $s / L \geq 2$, and the heating is fast, $\alpha t_{b} / s^{2} \leq 1$, where $L$ is the glass thickness, $s$ is the shaded edge width and $\alpha$ is the glass thermal diffusivity.
\end{abstract}

KEYWORDS: Window breaking, Glass temperatures, Compartment fire venting, Glass thermal stresses, Backdraft.

\section{INTRODUCTION}

Professor Emmons identified the problem of window breaking in compartment fires as an important unaddressed structural problem in his exemplar article on needed fire science at the First Symposium [1]. The mechanism he suggests for window breakage in fires is thermally induced tensile stress. All window glass has its surrounding edge covered by an opaque frame or gasket. Since glass is a relatively poor conductor, the edge remains unheated while the fire raises the temperature of the central portion by infrared radiation and hot gas convection. The thermal expansion of the uncovered window glass places the covered edge in tension until it cracks. Once the fracture begins, it bifurcates and very quickly propagates across the window; the glass falls out, creating a new vent in the compartment. Wired glass works, not by preventing fracture, but simply by holding the broken pieces in the frame and thereby avoiding a new vent. Double pane windows take longer to break than single pane because each pane sequentially undergoes the breaking process. Tempered glass also takes longer to break since the thermally created tension must first overcome the compression manufactured into tempered glass. 
In a review of fire physics at the Second Symposium [2], we suggested a simple strain criterion for the glass temperature increase, $\Delta T$, required to break windows in fires,

$\beta \Delta T=\sigma_{b} / E$,

where $\beta$ is the thermal coefficient of linear expansion, $\sigma_{b}$ is the tensile strength at breakage and $E$ is the Young's Modulus of the glass. Window (soda-lime) glass properties [3-6] are listed in Table 1 along with the corresponding strain, $\sigma / E$, and $\Delta T$ given by Eq.(1). The large range is due to the uncertainty in the $\sigma_{b}$ applicable to a particular window.

TABLE 1. Reported glass properties and calculated breaking strains and temperature increases in the limit $s / H \rightarrow 0$. The lower portion of the range is appropriate for typical window installations.

\begin{tabular}{|c|c|c|c|c|c|}
\hline Ref. No. & $\beta \times 10^{6}\left(K^{-1}\right)$ & $\sigma_{b} \times 10^{-7}\left(\mathrm{~N} / \mathrm{m}^{2}\right)$ & $E \times 10^{-10}\left(\mathrm{~N} / \mathrm{m}^{2}\right)$ & strain(\%) & $\Delta T(K)$ \\
\hline 3 & 9.5 & 4.7 & 7.0 & 0.07 & 70 \\
\hline 4 & 9.2 & $2.0-5.0$ & 7.2 & $0.03-0.07$ & $30-75$ \\
\hline 5 & 8.5 & $5.5-13.8$ & 7.24 & $0.08-0.19$ & $90-220$ \\
\hline 6 & 9.0 & $3.5-7.0$ & 7 & $0.05-0.10$ & $55-110$ \\
\hline
\end{tabular}

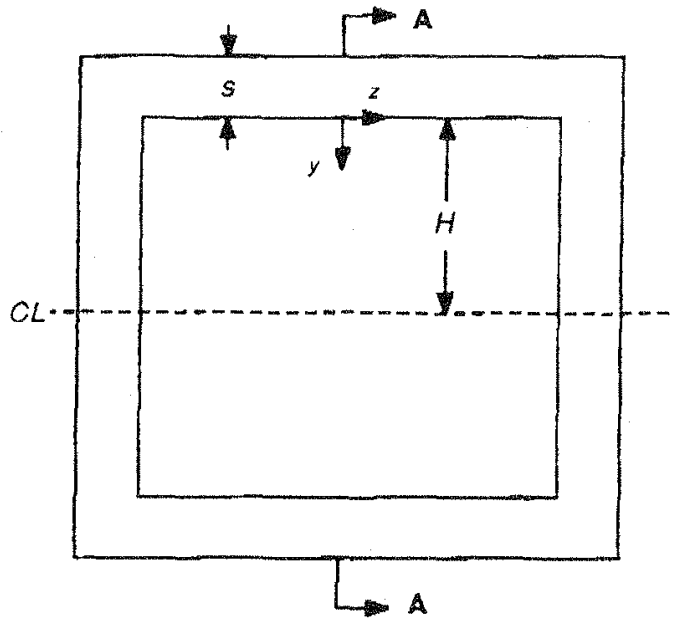

$1(\mathrm{a})$

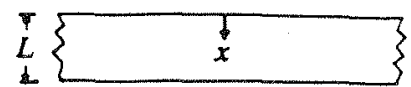

Section A-A

1(b)

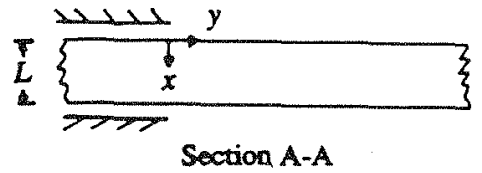

$1(c)$

Fig. 1. Window geometry. $x$ is the depth, $y$ is normal to the shaded region and $z$ is along the shading, $s$ is the width of the shading, $H$ is the half-length and $L$ is the glass thickness.

Figure 1 shows window glass schematics. Here $s$ is the shaded width under the frame, $H$ is the half-height of the unshaded window and $L$ is the glass thickness. In the case of large shading, $s / L \geq 2$, and fast heating, $\alpha t_{b} / s^{2} \leq 1$, this $\Delta T$ corresponds to the temperature difference between the initial temperature at the unheated outer edge and the transient temperature of the uniformly heated central section of the glass. Full-scale experimental confirmation of this method for predicting the time to window breakage, $t_{b}$, in compartment fires has been reported [3]. This method has also been applied to the reconstruction of fires where the breaking of windows has resulted in backdrafts [7]. Copius amounts of excess pyrolyzates had accumulated in the compartments. When the windows broke, fresh cold-air gravity currents mixed with the fuel-rich hot layer gases and migrated to the fires where the mixed layers were ignited. Turbulent flames then rapidly progressed through the accumulated pyrolyzates producing flashover. The conventional wisdom that windows break when the hot layer 
gas temperature reaches 530 to $650 \mathrm{~K}\left(500-700^{\circ} \mathrm{F}\right)$ [8] is a reasonable first approximation. This temperature could be specified in FIRST [9] to estimate the times at which windows become vents.

The purpose of this paper is two-fold: 1.) to quantify the connection between the compartment fire and the glass temperature and 2.) to provide detailed unsteady, multidimensional temperature and stress fields within the glass to extend our understanding beyond the simple strain criterion. The time to initial window breakage is our primary interest. We have not addressed the separate, but potentially important, question of the additional time required for all of the glass pieces to fall from the window frame [10].

In the next sections, several thermal analyses of the heating of glass by compartment fires, including the exponential decay of in-depth absorption of incident radiation and non-linear surface radiation, are presented. The stress fields produced by these temperature fields are then calculated. The article ends with suggestions for practical implementation.

\section{HEATED GLASS TEMPERATURE HISTORY, $\mathbf{T}(\mathbf{x}, \mathrm{t})$}

Consider the window shown in Fig. 1a and $1 \mathrm{~b}$. The goal is to calculate the temperature in the large central section of the glass as a function of depth into the glass, $x$, and time, $t$. Significant gradients, $\partial T / \partial x$, exist since the heat source is on the inside of the window and the sink is on the outside. All symbols are defined in the nomenclature. The unshaded glass is uniformly heated, so $\partial / \partial y$ and $\partial / \partial z$ are zero. The goveming equation is

$\rho c \frac{\partial T}{\partial t}=k \frac{\partial^{2} T}{\partial x^{2}}+I(t) \frac{e^{-x / l}}{l}$

where $I(t)$ is the incident radiative flux directly from the fire which is at sufficiently short wavelengths that its distributed intemal absorption needs to be included [11] and $l$ is the decay length in the glass (see e.g. Fig. 2 of Ref. 12). With the assumption that the glass is grey to other radiation, the initial and boundary conditions are

at $t=0, T=T_{i}$,

at $x=0,-k \frac{\partial T}{\partial x}=h_{2}\left(T_{2 \infty}(t)-T(0, t)\right)+\varepsilon_{\infty} \sigma T_{2 \infty}^{4}(t)-\varepsilon \sigma T^{4}(0, t)=q_{2}(t)$,

at $x=L,-k \frac{\partial T}{\partial x}=h_{1}\left(T(L, t)-T_{1 \infty}(t)\right)+\varepsilon \sigma T^{4}(L, t)-\varepsilon_{\infty} \sigma T_{1 \infty}^{4}(t)=q_{1}(t)$,

where side 1 is toward the ambient and side 2 is toward the hot layer in the compartment.

With the definitions

$\xi=\frac{x}{L} ; \tau=\frac{\alpha t}{L^{2}} ; \gamma=\frac{l}{L} ; \theta=\frac{T-T_{i}}{T_{c}} ; T_{c}=\sigma_{b} / E \beta ; \phi_{1}=\frac{q_{1}}{k T_{c} / L} ; \phi_{2}=\frac{q_{2}}{k T_{c} / L}$,

the dimensionless governing equation is

$$
\frac{\partial \theta}{\partial \tau}=\frac{\partial^{2} \theta}{\partial \xi^{2}}+j(t) \frac{e^{-\xi / \gamma}}{\gamma},
$$

with dimensionless initial and boundary conditions

at $\tau=0, \theta=0 ; \quad$ at $\xi=0,-\frac{\partial \theta}{\partial \xi}=\phi_{2}(\tau) ; \quad$ at $\xi=1,-\frac{\partial \theta}{\partial \xi}=\phi_{1}(\tau)$. 
Explicit expressions for the dimensionless heat fluxes, $\phi_{1}(\tau)$ and $\phi_{2}(\tau)$, are given in the Appendix. This equation is solved using a Laplace transform on time,

$\theta^{*}=\int_{0}^{\infty} \theta e^{-p \tau} d \tau$

The solution to the transformed problem is

$\theta^{*}=A_{1} j^{*} \frac{e^{-\xi / \gamma}}{\gamma}+B_{1} \cosh (\sqrt{p} \xi)+C_{1} \cosh (\sqrt{p}(1-\xi))$,

where

$A_{1}=\frac{1}{p-\frac{1}{\gamma^{2}}} ; B_{1}=\frac{A_{1} j^{*} e^{-1 / \gamma}-\gamma^{2} \phi_{1}^{*}}{\gamma^{2} \sqrt{p} \sinh \sqrt{p}} ; C_{1}=\frac{\gamma^{2} \phi_{2}^{*}-A_{1} j^{*}}{\gamma^{2} \sqrt{p} \sinh \sqrt{p}}$,

where $j^{*}, \phi_{1}^{*}$ and $\phi_{2}^{*}$ are the transformed fluxes. Regrouping Eq. (13) to explicitly identify the coefficients of those fluxes gives the transformed surface temperatures as

$\theta^{*}(0)=f_{1}{ }^{*}(0, p) \phi_{1}{ }^{*}+f_{2}{ }^{*}(0, p) \phi_{2}{ }^{*}+\frac{1}{\gamma} f_{3}{ }^{*}(0, p) j^{*}$

and

$\theta^{*}(1)=f_{1}{ }^{*}(1, p) \phi_{1}{ }^{*}+f_{2}{ }^{*}(1, p) \phi_{2}{ }^{*}+\frac{1}{\gamma} f_{3}{ }^{*}(1, p) j^{*}$,

where $f_{1}^{*}, f_{2}^{*}$ and $f_{3}^{*}$ are kernels which are functions of $\gamma, x$ and $p$ [13]. An advantage of this technique [14] is that the inversion need be performed only at the boundaries, to obtain $\theta(0, \tau)$ and $\theta(1, \tau)$. Using the convolution theorem and other simplifications, the equations to be solved are

$\theta(0, \tau)=\int_{0}^{\tau} \phi_{1}(\eta) f_{1}(0, \tau-\eta) d \eta+\int_{0}^{\tau} \phi_{2}(\eta) f_{2}(0, \tau-\eta) d \eta+\frac{1}{\gamma} \int_{0}^{\tau} j(\eta) f_{3}(0, \tau-\eta) d \eta$

and

$\theta(1, \tau)=\int_{0}^{\tau} \phi_{1}(\eta) f_{1}(1, \tau-\eta) d \eta+\int_{0}^{\tau} \phi_{2}(\eta) f_{2}(1, \tau-\eta) d \eta+\frac{1}{\gamma} \int_{0}^{\tau} j(\eta) f_{3}(1, \tau-\eta) d \eta$,

where $f_{1}, f_{2}$, and $f_{3}$ are kemels which are functions of $\gamma, x$ and $\tau$ [13]. These Volterra equations of the second kind are non-linear because the radiative parts of $\phi_{2}$ and $\phi_{1}$ are non-linear functions of $\theta(0, \tau)$ and $\theta(1, \tau)$. To avoid integrands which are unbounded as $\tau^{-1 / 2}$ at $\tau=0$, the transformation $u=\sqrt{\tau-\eta}$ is made. The final equations become

$\theta(0, \tau)=2 \int_{0}^{\sqrt{\tau}} u F_{1}(0, u) \phi_{1}\left(\tau-u^{2}\right) d u+2 \int_{0}^{\sqrt{\tau}} u F_{2}(0, u) \phi_{2}\left(\tau-u^{2}\right) d u+\frac{2}{\gamma} \int_{0}^{\sqrt{\tau}} u F_{3}(0, u) j\left(\tau-u^{2}\right) d u$

and

$\theta(1, \tau)=2 \int_{0}^{\sqrt{\tau}} u F_{1}(1, u) \phi_{1}\left(\tau-u^{2}\right) d u+2 \int_{0}^{\sqrt{\tau}} u F_{2}(1, u) \phi_{2}\left(\tau-u^{2}\right) d u+\frac{2}{\gamma} \int_{0}^{\sqrt{\tau}} u F_{3}(1, u) j\left(\tau-u^{2}\right) d u$,

where the $F_{i}(\xi, u)$ are evaluated from the $f_{i}(\xi, \tau)$ given in [13]. The numerical procedure chosen was a trapezoidal rule with constant time steps and thus variable $\Delta u$ since the $\phi_{i}$ are functions of $\theta$ which are known only at each time step. The Newton-Raphson method was used to find roots of the nonlinear equations.

Figure 2 shows the temperature history of the hot gas layer (calculated from FIRST [10] for a recent full scale fire) along with the surface temperatures given by Eqs. (18 and 19) from initiation to 
breakage. The $I=0$ case represents a window far from the fire; $I=10 \mathrm{~kW} / \mathrm{m}^{2}$ represents a window adjacent to the fire. The $100 s$ difference in $t_{b}$, defined by $\left[T\left(0, t_{b}\right)+T\left(L, t_{b}\right)\right] / 2=\sigma_{b} / E \beta$, allows the conditions in the compartment to change dramatically, as indicated by the hot layer temperature, $T_{2 \infty}(t)$. The temperature of the exposed side is sufficiently greater than that of the unexposed side to justify the detailed analysis of $T(x, t)$. Here $h_{2}, h_{1}$ and $I$ were taken as constants; the future compartment fire programs will hopefully provide these as functions of time. This section has quantified the coupling of the glass temperature to the compartment fire. The next section explores $T(x, y, t)$ to permit calculation of tensile stresses and quantification of geometric effects on $t_{b}$.

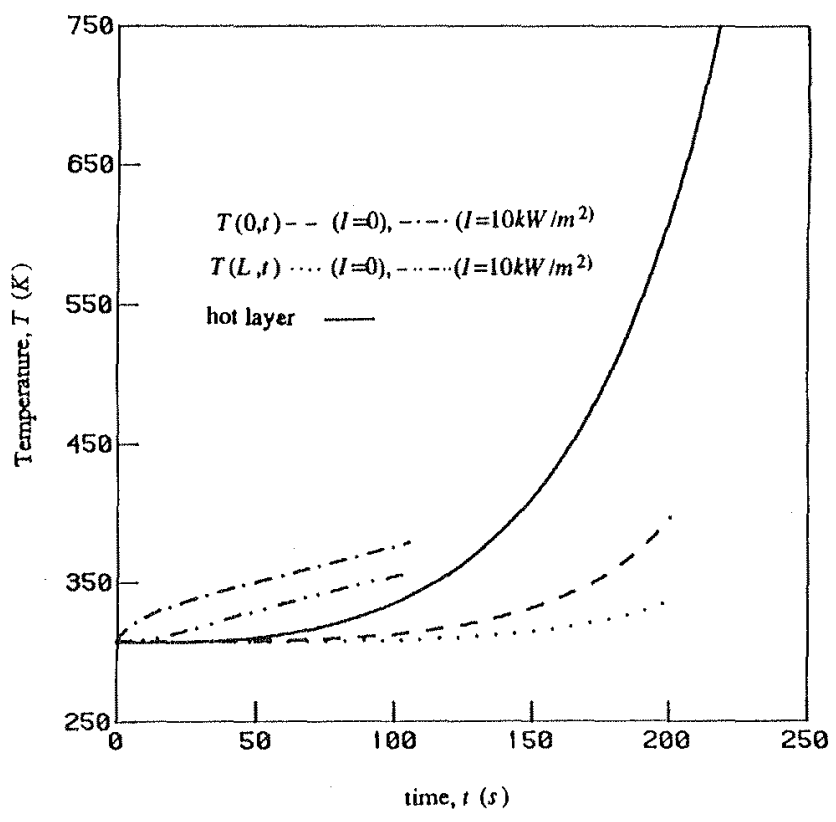

Fig. 2. Temperature history of the hot gas-layer and surface temperatures for $I(t)=0$ and $I(t)=$ $10 \mathrm{~kW} / \mathrm{m}^{2}$ with $\quad h_{2}=$ $50 \mathrm{~W} / \mathrm{m}^{2} K$ and $h_{1}=$ $10 \mathrm{~W} / \mathrm{m}^{2} \mathrm{~K}$.

\section{GLASS TEMPERATURE PROFILES, $T(x, y, t)$}

Consider now the glass pane section shown in Figure 1c. The range of interest is $-\infty<y+\infty$, so that the temperature field under the frame can be explored and the $s / L$ ratio at which the edge temperature increases can be determined. This range is reasonable since the frame and gasket have some non-zero conductivity. The origin of $y$ is on the inside edge of the frame. Here the goal is to calculate the temperature distribution along the pane from the shaded edge to the unshaded central section. The governing equation for this system is

$\rho c \frac{\partial T}{\partial t}=k\left[\frac{\partial^{2} T}{\partial x^{2}}+\frac{\partial^{2} T}{\partial y^{2}}\right]+\frac{l(t)}{l} e^{-x / l} H_{\nu}(y)$,

where $H_{\nu}$ is the Heaviside function and all other variables are as defined earlier. The initial condition and boundary conditions are 
$t=0, T=T_{i} ; \quad y \rightarrow \pm \infty \frac{\partial T}{\partial y}=0$

$x=0, \quad-k \frac{\partial T}{\partial x}=q_{2}(t) H_{v}(y) ; \quad x=L-k \frac{\partial T}{\partial x}=q_{1}(t) H_{v}(y)$.

The heat fluxes, $q_{2}(t), q_{1}(t)$ and $I(t)$ are assumed here to be specified apriori. Using the definitions given in Eq. (6), the governing equation is

$\frac{\partial \theta}{\partial \tau}=\frac{\partial^{2} \theta}{\partial \xi^{2}}+\frac{\partial^{2} \theta}{\partial \zeta^{2}}+\frac{j(\tau)}{\gamma} e^{-\xi / \gamma H_{\nu}(\zeta)}$

and the initial and boundary conditions are

$$
\begin{aligned}
& \tau=0, \theta=0 ; \quad \zeta \rightarrow \pm \infty, \frac{\partial \theta}{\partial \zeta}=0, \\
& \xi=0,-\frac{\partial \theta}{\partial \xi}=\phi_{2}(\tau) H_{v}(\zeta) ; \quad \xi=1,-\frac{\partial \theta}{\partial \xi}=\phi_{1}(\tau) H_{v}(\zeta) .
\end{aligned}
$$

In order to Fourier transform on $\zeta, \theta$ and $\partial \theta / \partial \zeta$ both have to approach zero as $\zeta$ approaches to. So the energy generation term and the fluxes are multiplied by $e^{-\delta \zeta}$ where $\delta$ is a small parameter. After obtaining the solution, the limit $\delta \rightarrow 0$ is taken. The Laplace transform is defined as in Eq. (11) and the Fourier transform is

$\overline{\theta^{*}}=\int_{-\infty}^{\infty} \theta^{*} e^{-i \omega \zeta} d \zeta$

The solution to the Fourier and Laplace transformed temperature as a function of $\xi, p$ and $\omega$ is given by

$\overline{\theta^{*}}(\xi, \omega, p)=\frac{p+\omega^{2}}{\delta+i \omega}\left[\phi_{1}^{*} f_{1}^{*}(p, \xi)+\phi_{2}^{*} f_{2}{ }^{*}(p, \xi)+\frac{i^{*}}{\gamma} f_{3}{ }^{*}(p, \xi)\right]$,

where $f_{1}{ }^{*}, f_{2}{ }^{*}$ and $f_{3}{ }^{*}$ are the same kernels as in Eq. (15) and $\phi_{1}{ }^{*}, \phi_{2}{ }^{*}$ and $j^{*}$ are the transformed fluxes. Applying the convolution theorem to invert the Fourier and Laplace transforms and taking the limit as $\delta \rightarrow 0$, gives the solution for the temperature field,

$\theta(\xi, \zeta, \tau)=\frac{1}{2} \int_{0}^{\tau}\left[\phi_{1}(\eta) f_{1}(\xi, \tau-\eta)+\phi_{2}(\eta) f_{2}(\xi, \tau-\eta)+\frac{1}{\gamma} j(\eta) f_{3}(\xi, \tau-\eta)\right]\left[1+\operatorname{erf}\left(\frac{\zeta}{\sqrt{4 \eta}}\right)\right] d \eta$

where the kernels $f_{1}(\xi, \tau), f_{2}(\xi, \tau)$ and $f_{3}(\xi, \tau)$ are the same as previously given. Here we again make a change of variable as $u=\sqrt{\tau-\eta}$ and evaluate the temperature field. The numerical integration procedure chosen was the trapezoidal rule with constant $\Delta u$ steps. Since the fluxes are known apriori, the calculation of the temperature field is simplified as iteration is no longer necessary.

Figure 3 shows dimensionless isotherms for the cases listed in the figure captions at times when the dimensionless surface temperature becomes 1 . The $q_{1}=0$ condition in Fig. 3a is clearly artificial, since the calculation gives $\theta(1, \tau)=0.4$ so that significant heat loss would occur to the ambient. However it represents a useful limit where the only heat sink is at $\zeta \rightarrow-\infty$, so the thermal penetration under the window frame is maximized. Fig. $3 \mathrm{~b}$ shows another example of that limit at a later time with gentler heating. Fig. $3 \mathrm{c}$ shows the effect of significant cooling at $\xi=1$. Figures $3 \mathrm{~b}$ and $\mathrm{c}$ represent conditions similar to Fig. 2. The slow fire in Fig. 3d produces a larger thermal penetration than $3 \mathrm{~b}$ or $3 \mathrm{c}$ because of its longer duration. 
Keski-Rahkonen did an excellent analysis [15] of the limiting case where: 1.) there are no temperature gradients across the glass thickness so a constant radiative input can be treated as a volumetric heat source; 2.) convective and linearized radiative heat loss is constant and identical on both sides of the glass so it can also be treated as a volumetric heat sink and 3.) all gas temperatures, the initial temperature and the temperature of the outer edge are constant and identical. His analytic result confirms Eq. (1) for most geometries of interest. Because of these restrictive heat flux assumptions, listed above, his analysis cannot be readily applied to compartment fires. However, it could describe small-scale experiments with a constant radiative flux [16].

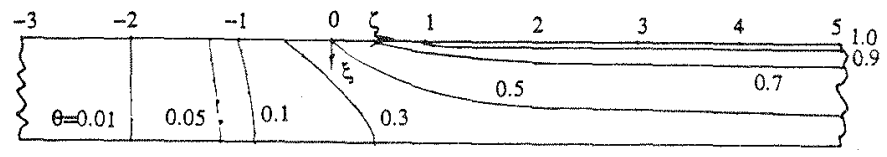

$3(\mathrm{a})$

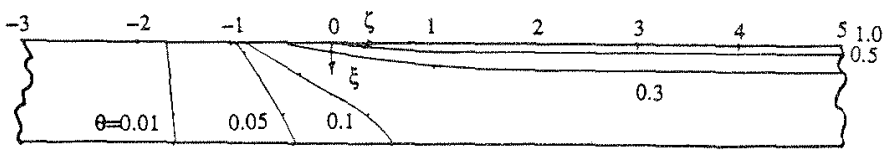

$3(\mathrm{~b})$

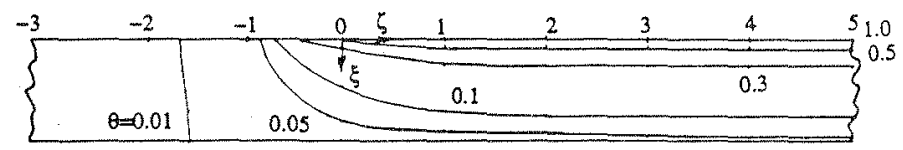

3(c)

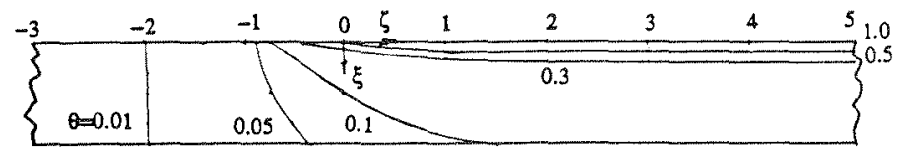

$3(d)$

Fig. 3. Two-dimensional temperature contours, $\theta=\left(T-T_{i}\right) /\left(\sigma_{b} / E \beta\right)$ for times at which $\theta(0, H / L, \tau)=1$, a: Fast fire next to the window, $\tau=0.66, q_{1}=0$, $I=5 \mathrm{~kW} / \mathrm{m}^{2}$ and $q_{2}=1 \mathrm{~kW} / \mathrm{m}^{2} \exp (t / 30 \mathrm{~s})$. b: Medium fire next to the window, $\tau=1,40, q_{1}=0, l=100 \mathrm{~W} / \mathrm{m}^{2} \exp (t / 30 s)$ and $q_{2}=200 \mathrm{~W} / \mathrm{m}^{2} \exp (t / 30 \mathrm{~s})$.

$c$ : Same as b, except $q \neq 0, \quad \tau=1.42, \quad q_{1}=0$ for $t<30 s$ and $100 \mathrm{~W} / \mathrm{m}^{2} \exp ((t-30 s) / 30 s)$ for $t \geq 30 s, I=100 \mathrm{~W} / \mathrm{m}^{2} \exp (t / 30 s)$ and $q_{2}=200 \mathrm{~W} / \mathrm{m}^{2} \exp (t / 30 \mathrm{~s})$. d: Slow fire away from the window, $\tau=3.17, q_{1}=0$ for $t<30 \mathrm{~s}$ and $10 \mathrm{~W} / \mathrm{m}^{2} \exp (((t-30 \mathrm{~s}) / 60 \mathrm{~s})$ for $t \geq 30 \mathrm{~s}, \quad I=0$ and $q_{2}=100 \mathrm{~W} / \mathrm{m}^{2}(t / 60 \mathrm{~s})$. 


\section{STRESS FIELD}

It is assumed that the temperature field is unaffected by the stress field and that the stresses instantaneously accommodate to changes in the temperature field. Since there are no $z$ temperature gradients, changes in stresses with $z$ are neglected. Let $\sigma_{z z}$ and $\sigma_{y y}$ be normal stresses in the $z$ and $y$ directions and $\sigma_{z y}$ and $\sigma_{y z}$ be the shear stresses on the $z$ and $y$ planes. All stresses are normalized on the tensile strength $\sigma_{b}, \psi=\sigma / \sigma_{b}$. To avoid a three dimensional stress analysis, we integrate over the glass thickness, $x$ [17]. Although significant temperature gradients have been shown, in the previous two sections, to exist across the window pane, the curvature these gradients produce does not affect the breaking stress since the window glass is assumed to be held in a flexible gasket. The dimensionless mean stresses are

$N_{z z}=\int_{0}^{1} \psi_{z z} d \xi ; \quad N_{y y}=\int_{0}^{1} \psi_{y y} d \xi ; \quad N_{y z}=N_{z y}=\int_{0}^{1} \psi_{y z} d \xi=\int_{0}^{1} \psi_{z y} d \xi$.

The local force balances in the $z$ and $y$ directions respectively are

$\frac{\partial N_{z z}}{\partial \mu}+\frac{\partial N_{z y}}{\partial \zeta}=0 ; \frac{\partial N_{y z}}{\partial \mu}+\frac{\partial N_{y y}}{\partial \zeta}=0$.

The boundary conditions are that there are no external forces acting on the plate and that the stress reaches a constant value near the center of the window,

$\int_{-s / L}^{H / L} N_{z z} d \zeta=0$ and $\frac{d N_{z z}}{d \zeta}=0$, as $\zeta \rightarrow \infty$.

The stress field is related to the temperature field by the compatibility relations [18]. These relations are obtained from the definitions of displacements and are basically relations for strain. The strains are then represented as stresses using the stress-strain relationships. After noting that $\partial / \partial z=0$ and defining the mean dimensionless temperature, $\left(\bar{T}-T_{i}\right) / T_{c}$, as

$N_{T}=\int_{0}^{1} \theta d \xi$

the compatibility equation simplifies to

$\frac{d^{2}}{d \zeta^{2}}\left[N_{z z}+N_{T}\right]=0$.

Integrating Eq. (37) twice and applying the boundary conditions, Eqs. (35), gives the stress throughout the range $-s / L<\zeta<H / L$, which by symmetry represents the entire window as,

$N_{z z}(\zeta, \tau)=\frac{L}{H+s} \int_{-s / L}^{H / L} N_{T}(\zeta, \tau) d \zeta-N_{T}(\zeta, \tau)$

Setting $N_{z z}\left(-s / L, \tau_{b}\right)=1$ in Eq. (38) shows that the mean temperature, $N_{T}$, not the surface temperature, $\theta(0, \tau)$, determines the time to breakage. Note however that it is still necessary to find $\theta(\xi, \tau)$, since the glass heat losses depend strongly on $\theta(0, \tau)$ and $\theta(1, \tau)$. The mean temperature may suffice for the stress history but it does not suffice to determine the temperature history. Figure 4 presents the mean stress from Eq.(38) as a function of $\zeta$ for the temperature fields given in Figure 3. For this case $s / L=3.0$ and $s / H=0.02$. The large tensile $(+)$ stress at the edge of the shaded region drops to a small compressive $(-)$ stress in the unshaded region. 


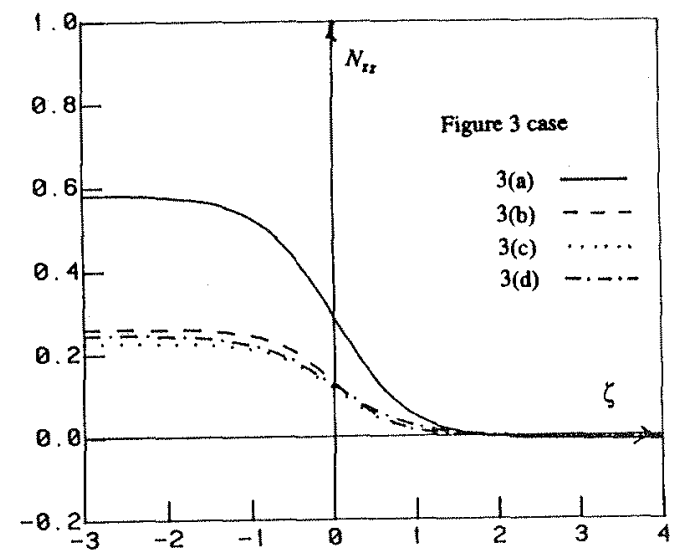

Fig. 4. Profiles of stress, $N_{z z}$, in the shaded, $\zeta<0$ and central, $\zeta>0$ regions for the temperature fields shown in Fig. 3. When any $N_{z z}$ reaches 1 , the glass breaks.

\section{APPLICATION}

Evaluating the dimensionless temperature field given by Eq. (32) for a large range of parameters [13] suggests that the variation of the mean temperature, $N_{T}$, with distance normal to the window's shaded edge, $\zeta$, can be well approximated, when the window is about to break, by a hyperbolic tangent (see Fig. 5). Therefore, we can reduce the application of Eq. (38) to simply the problem of predicting the window central temperature, if we assume a hyperbolic tangent profile and substitute

$N_{T}\left(\zeta, \tau_{b}\right)=\frac{g}{2}[1+\tanh \zeta]$ in $\quad 1=\frac{L}{H+s} \int_{-}^{H / L} N_{T}\left(\zeta, \tau_{b}\right) d \zeta-N_{T}\left(-s / L, \tau_{b}\right)$.

This yields a geometric factor $g$ which depends only on $s / L$ and $s / H$ given as

$g=2 /[\tanh (s / L)+\ln (\cosh (H / L) / \cosh (s / L)) L /(s+H)]$

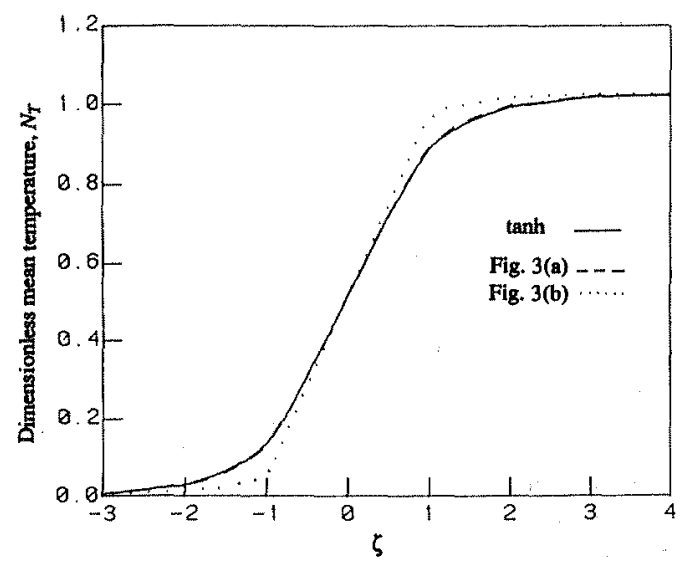

Fig. 5. Comparison of the $N_{T}$ profile at breakage, $N_{z z}=1$, with a hyperbolic tangent profile.

Figure 6 shows that $g$ remains near 1 for all reasonable $s / L$ and $s / H$. In the limiting case of a step temperature profile; $N_{T}=0$ for $\zeta<0$ and $N_{T}=g$ for $\zeta>0, N_{z z}=g /(1+s / H)$ for $\zeta<0$ and $-g /(1+H / s)$ for $\zeta>0$, so that $g=1+s / H$. The compression in the unshaded region is $1-g$. The variation in $g$ for the step and the hyperbolic tangent profile for $s / L \geq 2$ are quite close, indicating that the edge temperature remains close to the initial temperature (see Fig. 3). This suggests the 
criteria that the mean temperature at $-s$ can be taken as $T_{i}$, if $s / L \geq 2$ and $\alpha t_{b} / s^{2} \leq 1$, so that only the central temperature history, $T(x, t)$, obtained in Sec. 2 , is needed to find the glass breaking time. The correct approximate expression for the glass breaking time, $t_{b}$, is then

$$
\bar{T}\left(t_{b}\right)-T_{i}=g \sigma_{b} / E \beta
$$

where $\bar{T}\left(t_{b}\right)=\int_{0}^{L} T\left(x, H, t_{b}\right) d x / L$. This simply says the thickness-averaged temperature rise at the center of the window over that at the edge produces a thermal stress which exceeds the glass tensile strength. Typical values of the parameters in Eq.(41) suggest that the glass will break in a fire if $s / L \geq 2$. If these criteria are not met, the full temperature field, $T(x, y, t)$ as in Sec. 3 , is needed. The breaking time is then found from Eqs. (38, 36 and 32).

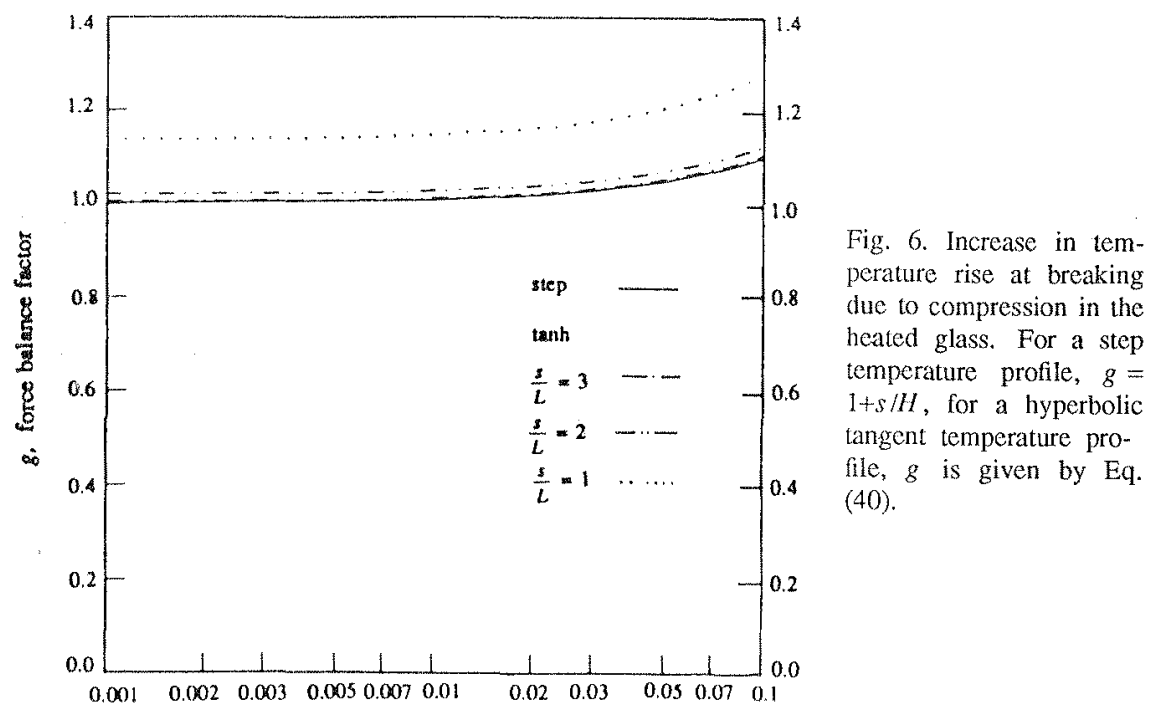

$s / H$, shaded length/half-width

Good compartment fire temperature histories are needed, as well as good estimates for convective and radiative heat transfer parameters to obtain the glass temperature, $T(x, t)$. In addition, Eq. (41) requires a value for window glass tensile breaking stress, $\sigma_{b}$. We have conducted a serics of experiments measuring $\sigma_{b}$ [13], which suggests a Weibull distribution for $\sigma_{b}$. This distribution implies that larger windows may break more easily since the probability of finding a low stress fracture initiation site increases with window area. Our data also suggest that for any size window the value of $\sigma_{b}=4 \times 10^{7} \mathrm{~Pa}(5800 \mathrm{psi})$ should be a good approximation to the lower limit of the breaking stress distribution. Perhaps the most fruitful approach to developing a glass which does not break in fires is to modify the glass formulation and manufacture to minimize the glass thermal expansion coefficient, i.e. design the glass so that $\beta \rightarrow 0$. Future work will include additional experimental comparisons and development of guidelines for selecting optimal convective, radiative and glass property input parameters. 


\section{ACKNOWLEDGEMENTS}

The sage advice of $H$. R. Baum was critical to the mathematical formulation and solution of this problem. G. C. Johnson assisted with the stress fields, I. Finnie with the glass properties and C. M. Fleischman with compartment fire temperatures. Support from the Building and Fire Research Laboratory at the United States National Institute for Standards and Technology under Grant 60 NANB8D0848 is very much appreciated.

\section{Nomenclature}

$\begin{array}{ll}A-G & \text { constants } \\ C & \text { specific heat } \\ E & \text { Young's modulus } \\ f, F & \text { kemels } \\ g & \text { geometry factor } \\ H & \text { half-length of the window } \\ H_{\nu}(y) & \text { Heaviside function } 0, \text { for } y \leq 0 \text { and } 1, \text { for } y>0 \\ h & \text { heat transfer coefficient } \\ I & \text { radiant heat flux directly from the flame } \\ j(t) & I(t) L / k T_{c} \\ k & \text { thermal conductivity } \\ L & \text { glass thickness } \\ l & \text { decay length } \\ q & \text { dimensional heat flux } \\ S & \text { shaded length } \\ T & \text { temperature } \\ T_{c} & \sigma_{b} / E \beta \\ \text { subscripts } \\ 1 & \text { compartment side of glass pane } \\ 2 & \text { ambient side of glass pane }\end{array}$

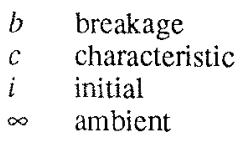

\section{Greek Characters}

$\alpha$ thermal diffusivity

$\beta \quad$ thermal expansion coefficient

$\gamma$ dimensionless decay length arbitrary small parameter emissivity dimensionless coordinate, $y / L$ dummy variable dimensionless temperature, $\left(T-T_{i}\right) / T_{c}$ dimensionless coordinate, $z / L$ stress dimensionless time, $\alpha t / L^{2}$ dimensionless coordinate, $x / L$ dimensionless heat flux, $q L / k T_{c}$

$\psi$ dimensionless stress, $\sigma / \sigma_{b}$

\section{REFERENCES}

1. Emmons, H. W. "The Needed Fire Science", Fire Safety Science-Proceedings of the First International Symposium, eds. C. E. Grant and P. J. Pagni, pp. 33-53, Hemisphere Washington, D.C., 1986.

2. Pagni, P. J. "Fire Physics-Promises, Problems and Progress", Fire Safety Science-Proceedings of the Second International Symposium, eds. T. Wakamastu et. al. pp. 49-66, Hemisphere, Washinton, D.C., 1988.

3. Skelly, M. J. "An Experimental Investigation of Glass Breakage in Compartment Fires", M. S. Thesis, Virginia Polytechnic Institute., 1990.

4. Bansal, N. P., and Doremus, R. H., Handbook of Glass Properties, pp. 363, 368, Academic Press Inc., New York, N. Y., 1986.

5. McLellan, G. W. and Shand, E. B., Glass Engineering Handbook, pp. 2-16, 2-18, McGraw Hill Book Co., New York, N. Y., 1984.

6. Fletcher, D. Q., Mechanics of Materials, p 474, Holt, Rinehart and Winston, New York, N. Y., 1985.

7. Pagni, P. J. and Fleischmann C. M., private communication.

8. Jensen, R., private communication. 
9. Mitler, H. E., and Rockett, J. A., "User's Guide to FIRST, A Comprehensive Single-Room Fire Model", Report No.87-3595, p. 124, National Institute of Standards and Technology, Gaithersburg, MD, 1987.

10. Emmons, H. W., "Window Glass Breakage by Fire", Home Fire Project Technical Report No. 77, Harvard University, Cambridge, MA, 1988.

11. Lund, P. J., Solar Thermal Engineering, p. 126, John Wiley and Sons, New York, N. Y., 1980.

12. Gardon, R., "A Review of Radiant Heat Transfer in Glass", Journal of The American Ceramic Society, 44, 305-313, 1961.

13. Joshi, A. A., and Pagni, P. J., "Fire Induced Thermal Fields in Window Glass", submitted to Fire Safety Journal.

14. Chambres, P. L., "Nonlinear Heat Transfer Problem", Journal of Applied Physics, 30, 1683-1688, 1959.

15. Barth, P. K. and Sung, H., "Glass Fracture under Intense Heating", Senior course project, Harvard University, Cambridge, MA, 1977.

16. Keski-Rahkonen, O., "Breaking of Window Glass Close to Fire", Fire and Materials, 12, 61-69, 1988.

17. Boley, B. A., and Weiner, J. H., Theory of Thermal Stresses, R. E. Krieger Publishing Company, Malabar, FL, 1985.

18. Wang, C., Applied Elasticity, p. 22, McGraw-Hill, New York, N. Y., 1953.

19. Joshi, A. A. and Pagni, P. J., "User's Guide to BREAK1, The Berkeley Algorithm for Breaking Window Glass in a Compartment Fire", submitted to NIST.

\section{APPENDIX}

The heat fluxes in Eqs. (9) and (10) are

$\phi_{2}(t)=A+B \theta(0, \tau)+C \theta^{2}(0, \tau)+D \theta^{3}(0, \tau)+E \theta^{4}(0, \tau)$

and

$\phi_{1}(\tau)=F+G \theta(1, \tau)-C \theta^{2}(1, \tau)-D \theta^{3}(1, \tau)-E \theta^{4}(1, \tau)$

where

$A=\frac{h_{2} L\left(T_{2 \infty}(t)-T_{i}\right)}{k T_{c}}+\frac{\varepsilon_{\infty} \sigma L T_{2 \infty}{ }^{4}(t)}{k T_{c}}-\frac{\varepsilon \sigma L T_{i}{ }^{4}}{k T_{c}} ; B=-\frac{h_{2} L}{k}+\frac{4 \varepsilon \sigma T_{i}{ }^{3} L}{k}$

$C=-\frac{6 \varepsilon \sigma T_{c} T_{i}^{2} L}{k} ; D=-\frac{4 \varepsilon \sigma T_{c}^{2} T_{i} L}{k} ; E=-\frac{\varepsilon \sigma^{\prime} T_{c}^{3} L}{k}$

$F=\frac{h_{1} L\left(T_{i}-T_{1 \infty}(t)\right)}{k T_{c}}-\frac{\varepsilon_{\infty} \sigma T_{1 \infty}^{4}(t) L}{k T_{c}}+\frac{\varepsilon \sigma L T_{i}^{4}}{k T_{c}} ; G=\frac{h_{1} L}{k}+\frac{4 \varepsilon \sigma T_{i}^{3} L}{k}$ 